\title{
Sonographic Evaluation of Early-Stage Breast Cancers That Undergo Neoadjuvant Chemotherapy
}

\author{
Marilyn A. Roubidoux, MD, Gerald L. LeCarpentier, PhD, \\ J. Brian Fowlkes, PhD, Brett Bartz, BS, Deepa Pai, MS, BS, \\ Sasha P. Gordon, BS, RDMS, Anne F. Schott, MD, Timothy D. \\ Johnson, PhD, Paul L. Carson, PhD
}

\begin{abstract}
Abbreviations
BIRADS, Breast Imaging Reporting and Data System; $\mathrm{MRI}$, magnetic resonance imaging; PET, positron emission tomography; ROI, region of interest; SWD, speedweighted pixel density; 3D, 3-dimensional
\end{abstract}

Received December 20, 2004, from the Departments of Radiology (M.A.R., G.L.L., J.B.F., P.L.C.) and Internal Medicine (A.F.S.), University of Michigan Health Systems, Ann Arbor, Michigan USA; University of Michigan School of Medicine, Ann Arbor, Michigan USA (B.B.); Department of Biostatistics, University of Michigan, Ann Arbor, Michigan USA (T.D.J.); Oakwood Healthcare System, Dearborn, Michigan USA (D.P.); and Primary Children's Medical Center, Salt Lake City, Utah USA (S.P.G.). Revision requested January 25, 2005. Revised manuscript accepted for publication February 17, 2005.

This work was supported in part by National Institutes of Health grant RO1 CA 91713.

Address correspondence to Marilyn $A$. Roubidoux, MD, Department of Radiology, University of Michigan Health Systems, 2910G Taubman Center, Ann Arbor, MI 48109-0326 USA.
Objective. We prospectively evaluated low-stage breast cancers treated with neoadjuvant chemotherapy using whole-volume sonography and color Doppler imaging. Methods. Thirty-four women with breast cancer (mean maximum size, $2.4 \mathrm{~cm}$ ) received neoadjuvant chemotherapy with doxorubicin and docetaxel. Targeted whole-volume sonography of tumor sites was performed before and after chemotherapy to assess mass size, color pixel speed-weighted density, and American College of Radiology Breast Imaging Reporting and Data System sonographic characteristics. After chemotherapy, tumor sites were excised by lumpectomy or mastectomy. Results. Three $(11.3 \%)$ of 34 patients had a complete histologic response. After chemotherapy, correlation was $r=0.716$ between final histologic and sonographic sizes. Compared with histologic residual tumors, sonography had 4 false-negative results, 3 false-positive results, and 27 true-positive results (sensitivity, 87\%), with no false-negative results among a subgroup of tumors of $7 \mathrm{~mm}$ and larger (sensitivity, 100\%). The 3 cases with false-positive results were histologic fibrosis or biopsy changes. Mean speed-weighted density was 0.015 before and 0.0082 after chemotherapy $(P=.03)$. After chemotherapy, vascularity was less common within $(P=.06)$ or adjacent to $(P=.009)$ masses or in tumor sites $(P=.05)$. Prechemotherapy variables of gray scale characteristics and vascularity were compared with final histologic size, and all had $P>.20$. Conclusions. Postchemotherapy sensitivity of sonography was high for residual tumors of $7 \mathrm{~mm}$ or larger. Correlation was moderate between histologic and sonographic final tumor sizes. False-positive results were caused by fibrosis or biopsyrelated changes. False-negative results occurred with residual tumor size of $6 \mathrm{~mm}$ or smaller. After chemotherapy, vascularity usually decreased, and this was not specific for complete response. Before chemotherapy, no vascular or gray scale feature at initial imaging predicted complete responders. Key words: breast cancer; breast sonography; neoadjuvant chemotherapy.

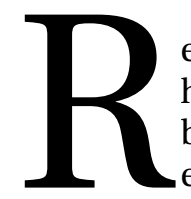

ecent clinical trials of women with breast cancer have shown that treatment with chemotherapy before definitive surgery (neoadjuvant) results in equal survival overall when compared with a surgery-first treatment plan. By reducing tumor bulk before definitive surgery, neoadjuvant chemotherapy provides the opportunity for breast conservation and for 
a smaller excision size. ${ }^{1,2}$ In addition to improving the rates of breast conservation, a complete pathologic response to neoadjuvant chemotherapy has been shown to be a reliable predictor of overall survival. ${ }^{1,2}$ Furthermore, access to the primary tumor during early treatment allows for in vivo testing and allows for evaluation of chemoresponsiveness of the patient's breast cancer.

However, the selection of candidates for lumpectomy after completion of chemotherapy is not straightforward, and the assessment of tumor response can be difficult. Accurate, readily available, and cost-effective clinical imaging techniques to assess tumor extent after neoadjuvant therapy or to predict response at initial evaluation would greatly assist clinical care. ${ }^{1}$ Physicians use palpation, mammography, magnetic resonance imaging (MRI), sonography, and positron emission tomography $(\mathrm{PET})^{1,3-7}$ to assess the clinical response to neoadjuvant chemotherapy, but results may be conflicting. For example, the physical examination often suggests a complete therapeutic response, whereas the mammogram shows extensive residual calcifications, ${ }^{7}$ which may be suggestive of remaining cancer.

Neoadjuvant chemotherapy is commonly used to treat locally advanced cancers, but the current trend is to extend the use of neoadjuvant treatment to T1 tumors. ${ }^{2}$ Many previous studies among locally advanced breast cancers have evaluated the ability of mammography, MRI, sonography, and PET to distinguish the pathologic response to neoadjuvant chemotherapy. ${ }^{3-7}$ One study also reported that PET could even predict the response to chemotherapy on the basis of evidence that tumor blood flow and metabolism were predictors of response. ${ }^{8}$

To our knowledge, there are no reports of imaging studies targeted to low-stage ( $\mathrm{T} 1$ and T2) breast cancers that undergo neoadjuvant chemotherapy. As neoadjuvant chemotherapy becomes a more widespread treatment, the role of breast sonography should be elaborated. We studied lower-stage breast cancers using wholevolume sonography augmented with color Doppler imaging. Our purposes were to assess the accuracy of sonography in detecting residual primary breast malignancy, to evaluate how gray scale and color Doppler characteristics of tumors change during chemotherapy, and to determine whether any of these characteristics were predictive of response to chemotherapy.

\section{Materials and Methods}

\section{Patient Group}

Patients were recruited from a multidisciplinary breast care center at a national comprehensive cancer center into a trial of neoadjuvant chemotherapy. This prospective study evaluated patients who had early-stage operable breast cancers that were found between January 11, 1999, and August 12, 2002. Institutional Review Board approval and informed consent were obtained. Eligibility criteria included women with core needle biopsy-proved invasive cancers that were T1, T2, or T3, N0, N1, or N2, and M0. Women with T4 tumors were excluded. Each woman was treated with doxorubicin and docetaxel as neoadjuvant chemotherapy, after which the tumor sites were excised by lumpectomy, or mastectomy specimens were histologically processed and interpreted in the customary way for breast lesions at our institution. The chemotherapy consisted of doxorubicin $\left(50 \mathrm{mg} / \mathrm{m}^{2}\right)$ and docetaxel $\left(75 \mathrm{mg} / \mathrm{m}^{2}\right)$ on day 1 , every 3 weeks, for 4 cycles during a 16-week period. Histologic findings were obtained from medical records.

Forty-one women underwent whole-volume breast sonography including gray scale and color Doppler imaging of the primary cancer both before and after chemotherapy. Six patients were excluded from analysis because their tumors appeared larger than $4 \mathrm{~cm}$ (transducer width), which made it technically difficult to assess the vascularity and gray scale features from a single transducer sweep. One patient was excluded from analysis because the tumor was incompletely scanned and only partially visible in the image volume. Thus, the study group consisted of 34 women (age range, 27-61 years; mean age, 45 years) with invasive cancers of 1 to $4 \mathrm{~cm}$ (mean maximum tumor size, $2.4 \mathrm{~cm}$ ).

\section{Sonographic Image Acquisition}

A sonographic examination of each patient was performed with a GE LOGIQ 700 scanner (GE Healthcare, Waukesha, WI) using an M12 linear matrix array scan head (6 MHz Doppler and 9 $\mathrm{MHz}$ gray scale). The region that contained the tumor was scanned, and, on average, this measured approximately $4 \mathrm{~cm}$ in width by 3.5 to $6 \mathrm{~cm}$ in length. Scanning was performed in a manner previously described by Bhatti et $\mathrm{al}^{9}$ and LeCarpentier et al. ${ }^{10}$ The images were acquired at approximately 0.5 -mm intervals in gray scale and 
color Doppler modes. With the use of a compact hand-driven scanner, the images were acquired at approximately $0.5-\mathrm{mm}$ intervals in gray scale and color Doppler modes and interfaced to a personal computer. To identify the location of the mass for sonographic imaging before and after chemotherapy, multiple sources of information were used, including palpation, clinically obtained mammographic and sonographic images and their reports, markers on the skin (tattoo), and biopsy clips. In addition, the exact location of the mass, the scan direction, and sonographic settings were recorded at the initial prechemotherapy research sonographic scans, and then the scans were performed in the same manner after completion of chemotherapy. The research sonographic scans were performed by 1 technologist experienced in breast sonographic imaging, also trained in mammography. The skin over the breast mass was coated with gel, and the breast was stabilized by stretching a 2-mil polyethylene film (also coated with gel) across the chest to decrease tissue motion. For color Doppler imaging, the patient's electrocardiogram was used to trigger the foot switch of the GE scanner. Thus, cardiac gating was used for image capture during systole, which maximized the Doppler signal.

\section{Image Post Processing}

Sixty to 90 images per scan were stored in the cine loop and then transferred to a workstation for post processing. The scan slices were stacked to render a 3-dimensional (3D) volume. Each 3D volume was displayed in AVS/Express data visualization software (Advanced Visual Systems, Waltham, MA) as a series of 3 intersecting planes. In-house tools designed with AVS/Express allowed a radiologist to review the image volumes and slices and to determine the margins in 3 dimensions of each mass. The radiologist (M.A.R.) selected a 3D region of interest (ROI) that identified the mass in each tissue volume. To delineate the ROI from the surrounding tissue, a dynamically positioned and shaped ellipsoidal $3 \mathrm{D}$ volume was formed by the AVS/Express software, a procedure described previously. ${ }^{9,10}$ These ROIs were placed prospectively in the image sets before the final histologic results at excision were available. Therefore, the radiologist was blinded to final histologic outcome at the time of identification of the masses. The prechemotherapy sonographic images were used to help assess the postchemotherapy images in each patient.

\section{Image Analysis}

\section{Mass Size and Volumes}

These were calculated from the ROIs by in-house software.

\section{Mass Characterization}

The whole-volume gray scale image sets were randomized, and readers were blinded to patient information and dates of the scans. Characteristics of the masses were classified according to the American College of Radiology Breast Imaging Reporting and Data System (BIRADS) lexicon sonographic categories ${ }^{11}$ and were read by consensus by 2 readers (M.A.R. and S.P.G.). The shapes of the masses were classified as oval, round, or irregular. Orientation was classified as either parallel or not parallel. Margins were classified as either circumscribed or not circumscribed. The lesion boundary was classified as either an abrupt interface or echogenic. Echogenicity was classified as complex, hypoechoic, or isoechoic (no cases were either anechoic or hyperechoic). Posterior acoustic features were classified as no posterior acoustic features, enhancement, shadowing, or a combined pattern. The ratings for all scans were recorded in a Microsoft Excel (Microsoft Corporation, Redmond, WA) spreadsheet and tabulated.

\section{Color Doppler Assessments}

Subjective Measures. By the same readers above, vascularity was classified from the color Doppler image volume according to the 4 BIRADS sonographic vascularity categories: "not present" (defined as in or adjacent to a mass), "present in the lesion," "present immediately adjacent to the lesion," and "diffusely increased vascularity in surrounding tissue." ${ }^{11}$ If vascularity was present, 1 or more of the other 3 classifications could be present. Thus, the last 3 categories were not mutually exclusive.

Quantitative Measures. The vascularity of each mass was assessed from the ROI in the color Doppler image sets by a quantitative computer method previously described using a formula designated as speed-weighted pixel density (SWD). ${ }^{9,10}$ This formula measures the density of color pixels in a gray scale volume.

Differences between before and after chemotherapy for these vascularity assessments were analyzed by the $t$ test (for SWD values) and McNemar test (for subjective ratings). 


\section{Data Analysis}

To determine the accuracy of sonography in detecting residual tumors after chemotherapy, sonographic findings after chemotherapy (ie, presence of a mass and its maximal size) were compared with histologic reports at final excision by lumpectomy or mastectomy (ie, presence of malignant tumor and its maximal size). Each postchemotherapy sonographic result was then categorized as true-positive, true-negative, falsepositive, or false-negative. The final histologic size was compared with the postchemotherapy sonographic size with the use of a correlation coefficient. Final histologic size was compared with the prechemotherapy subjective classifications (BIRADS sonographic categories) of the gray scale and color Doppler characteristics and with the color Doppler SWD values to determine whether any feature predicted response to chemotherapy. Categorical variables were analyzed with 1-way analysis of variance, and continuous variables were analyzed with linear regression.

\section{Results}

\section{Histologic Results at Final Excision Compared With Postchemotherapy Sonographic Results} After completion of chemotherapy, 3 of the 34 patients had no residual invasive or intraductal cancer at histologic examination (ie, complete response), and 31 patients had residual cancer (maximal size range, $0.2 \mathrm{~cm}$ of ductal carcinoma in situ to $3.5 \mathrm{~cm}$ of invasive cancer; mean, 1.2 $\mathrm{cm})$. Therefore, compared with mean maximum size by sonography before chemotherapy $(2.4 \mathrm{~cm})$, there was, on average, a $50 \%$ decrease in maximum tumor size. The postchemotherapy mean maximum size by sonography in the entire group (34 cases) was $1.2 \mathrm{~cm}$, which was the same as the final mean maximum histologic size of $1.2 \mathrm{~cm}$. The maximum sonographic size of the masses was compared with the maximum histologic size, and these values are summarized in Figure 1. There was moderate correlation between the sonographic values and the histologic values $(r=0.716 ; P \leq .002)$. This graph also depicts the cases that had sonographic false-negative (4), false-positive (3), and true-positive (27) results. Three cases had sonographic masses at the end of chemotherapy, without residual histologic tumors (the complete responders); therefore, these were the 3 cases with false-positive sonographic results. The histologic reports for these cases indicated the presence of fibrosis (2 cases) and biopsyrelated change (1 case) (Figure 2, A and B). Because no sonographic mass was identifiable after chemotherapy despite histopathologic evidence of tumor cells, 4 cases had false-negative results; all were $6 \mathrm{~mm}$ or smaller in maximum histologic size.

A subgroup of cases with a maximum histologic size of residual cancer of $0.7 \mathrm{~cm}$ or larger (23 cases) all had a sonographic mass. Therefore, there were no false-negative sonographic results in this subgroup. The sonographic size was within $1 \mathrm{~cm}$ of the final histologic size in $28(82 \%)$ of 34 patients. Sonography underestimated the extent of residual cancer by greater than $1 \mathrm{~cm}$ in $2(6 \%)$ patients and overestimated it by greater than $1 \mathrm{~cm}$ in $4(12 \%)$ patients, 2 of whom had the false-positive results (complete responders). In 1 of these cases, the pathology report indicated that the exact size was very difficult to determine. In the cases with true-positive results, the initial mean volume was $6475 \mathrm{~mm}^{3}$ and the final mean volume was $1342 \mathrm{~mm}^{3}$ (79\% decrease).

Figure 1. Postchemotherapy mass size by sonography compared with histologic size. Diamonds indicate true-positive; squares, false-positive; and triangles, false-negative $(r=0.716 ; P \leq .002)$.

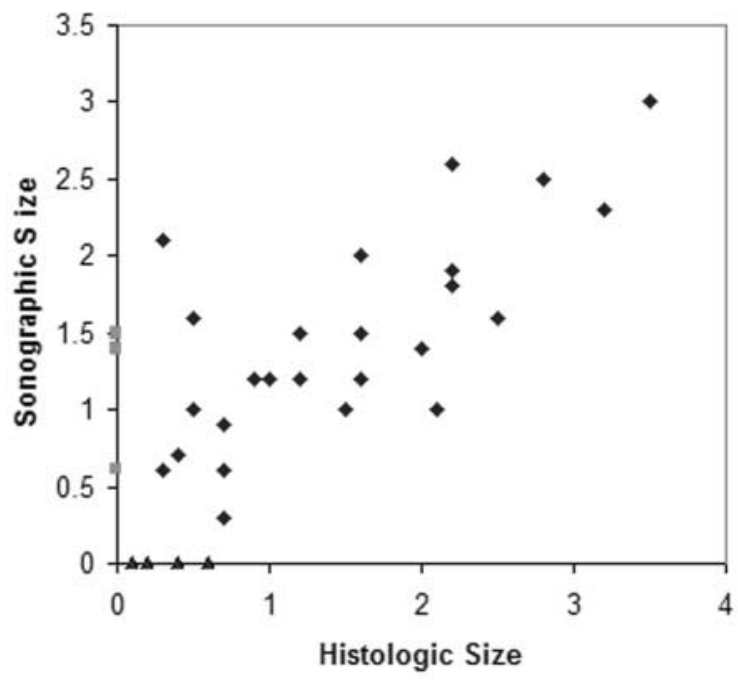




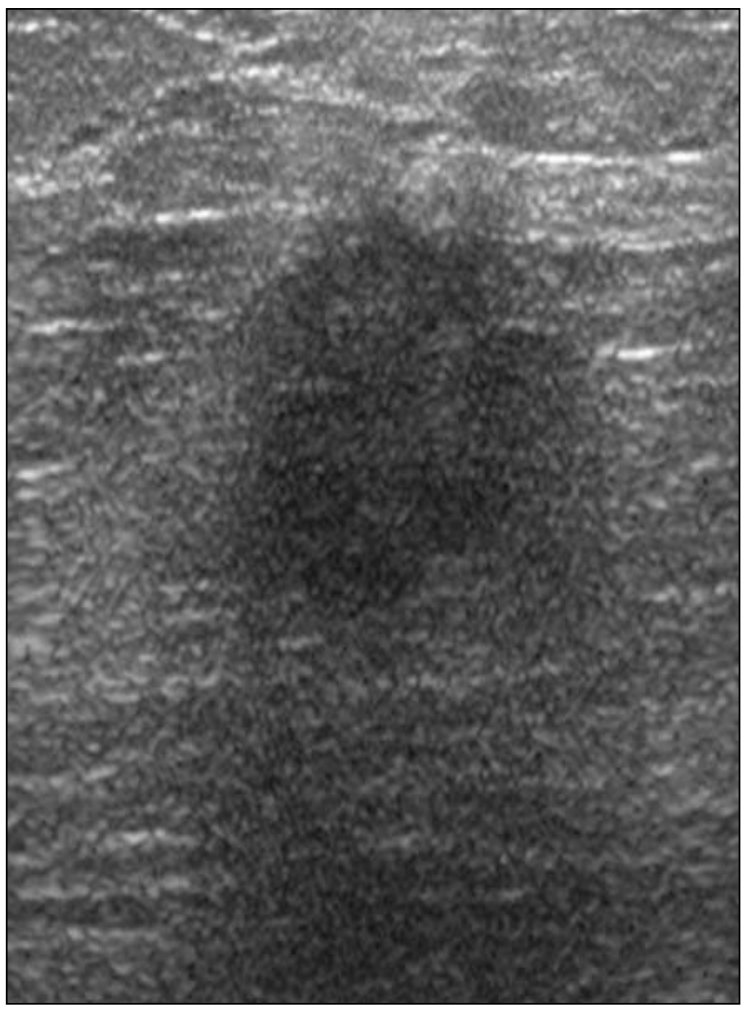

A

Figure 2. Histologic complete response. A, Before chemotherapy, the breast cancer is a taller-than-wide hypoechoic mass with indistinct margins and slight shadowing. B, After, chemotherapy, there is a smaller hypoechoic oval mass with indistinct margins, which simulated residual malignancy and was fibrosis at final histologic examination.

\section{Changes in Mass Characteristics and Color Doppler Signal During Chemotherapy}

\section{Mass Characteristics}

The BIRADS gray scale characteristics are summarized in Table 1, grouped by before and after chemotherapy and according to true-positive, false-positive, and true-negative results. Among the 27 cases with true-positive results (those having a residual tumor at final excision and a sonographic mass after chemotherapy), 26 were evaluated (1 case was excluded from this analysis because 1 computer file was lost at the time of analysis). Overall, 20 (77\%) of 26 masses changed in 1 gray scale characteristic or more, whereas 6 $(24 \%)$ did not change in any gray scale feature. The most common change was in posterior acoustic features (12 cases), and change in shape was occasionally dramatic (Figures 3, A and B, and 4, A and B). There was no consistent pattern of change among the BIRADS sonographic features. There were no gray scale features that were useful to distinguish false-positive from true-positive results.

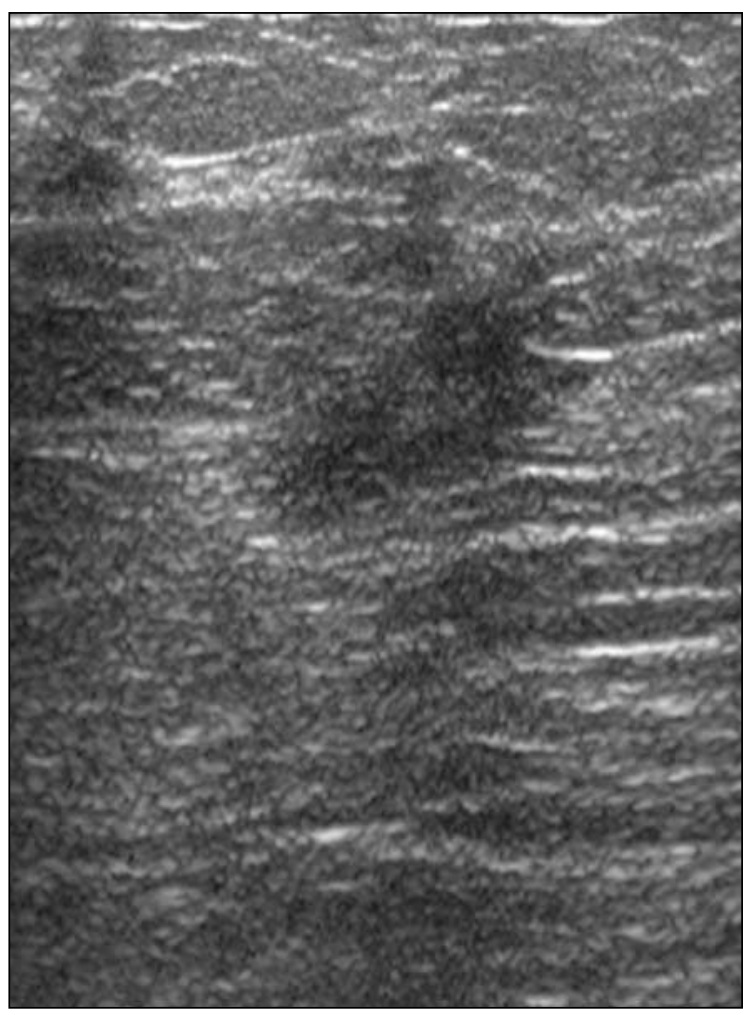

B 
It should be observed that changes in these SWD values do not reflect volume or size changes because the SWD measure is the rate of color pixels to gray scale pixels in a given volume. Therefore, a change in the vascularity of a mass as measured by SWD is independent of a change in the size of a mass.

The subjective ratings before and after chemotherapy are summarized in Table 3 . This shows that the color Doppler signal (ie, vascularity) significantly decreased after chemotherapy in 3 of the 4 categories, and after chemotherapy, half of the cases had absent vascularity.

Before chemotherapy, neither subjective vascularity ratings nor the SWD values appeared to correlate to the final histologic size or to a complete histologic response. For example, among the 6 cases that initially had absent vascularity, the final histologic mean size was $1.4 \mathrm{~cm}$ (range, $0.7-3.2 \mathrm{~cm}$ ), and among the 6 cases with the highest vascularity, the final histologic mean size was $1.5 \mathrm{~cm}$ (range, $0-3.5 \mathrm{~cm}$ ). Thus, initial vascularity did not predict the tumor response to chemotherapy. It may be noted that the 3 cases that had a complete histologic tumor response (no residual cancer) had at initial imaging comparatively low SWD values and no vascularity in the lesion. However, because of small numbers of cases that were complete responders, this was not statistically significant. When the quantitative and subjective vascularity characteristics were compared with final histologic size, $P$ values were not significant and ranged from .27 to .70 .

At the end of chemotherapy, among the 12 masses with absent vascularity subjectively and objectively, 2 had no residual cancer, 1 had 0.2 $\mathrm{mm}$ of ductal carcinoma in situ, and 9 had residual invasive cancer (mean maximum size, 1.1 $\mathrm{cm}$; range $0.1-2.8 \mathrm{~cm}$ ). Therefore, the absence of vascularity at the end of chemotherapy was common and was not specific for a complete response.

\section{Discussion}

Neoadjuvant chemotherapy increases the chance of breast conservation, decreases the amount of excised breast tissue necessary for treatment, and allows the observation of the chemoresponsiveness of breast cancers. ${ }^{1,2,12}$ The

Table 1. Sonographic BIRADS Categories of the Sonographic Masses

\begin{tabular}{|c|c|c|c|c|c|c|}
\hline \multirow[b]{2}{*}{ Characteristic } & \multicolumn{2}{|c|}{$\begin{array}{c}\text { True-Positive } \\
(\mathrm{n}=26)\end{array}$} & \multicolumn{2}{|c|}{$\begin{array}{c}\text { False-Positive* } \\
(n=3)\end{array}$} & \multicolumn{2}{|c|}{$\begin{array}{l}\text { False-Negative } \\
\quad(n=4)\end{array}$} \\
\hline & Before & After & Before & After & Before & After \\
\hline \multicolumn{7}{|l|}{ Margins } \\
\hline Circumscribed & 11 & 9 & 2 & 0 & 0 & NA \\
\hline Not circumscribed & 15 & 17 & 1 & 3 & 4 & NA \\
\hline \multicolumn{7}{|l|}{ Shape } \\
\hline Round & 3 & 1 & 2 & 0 & 0 & NA \\
\hline Oval & 13 & 16 & 1 & 2 & 3 & NA \\
\hline Irregular & 10 & 9 & 0 & 1 & 1 & NA \\
\hline \multicolumn{7}{|l|}{ Orientation } \\
\hline Parallel & 12 & 15 & 1 & 2 & 1 & NA \\
\hline Not parallel & 14 & 11 & 2 & 1 & 3 & NA \\
\hline \multicolumn{7}{|l|}{ Echo pattern } \\
\hline Complex & 3 & 2 & 0 & 0 & 0 & NA \\
\hline Hypoechoic & 19 & 18 & 2 & 2 & 4 & NA \\
\hline Isoechoic & 4 & 6 & 1 & 1 & 0 & NA \\
\hline \multicolumn{7}{|c|}{ Posterior acoustic features } \\
\hline None & 4 & 14 & 0 & 2 & 2 & NA \\
\hline Enhancement & 9 & 4 & 1 & 0 & 1 & NA \\
\hline Shadowing & 9 & 8 & 2 & 1 & 1 & NA \\
\hline Combined & 4 & 0 & 0 & 0 & 0 & NA \\
\hline \multicolumn{7}{|l|}{ Lesion boundary } \\
\hline Abrupt & 16 & 18 & 2 & 3 & 2 & NA \\
\hline Echogenic & 10 & 8 & 1 & 0 & 2 & NA \\
\hline
\end{tabular}

After indicates after completion of the course of chemotherapy; Before, before administration of chemotherapy; and NA, not applicable.

*At final histologic examination, there was no residual tumor, although a mass was visible on sonography. 


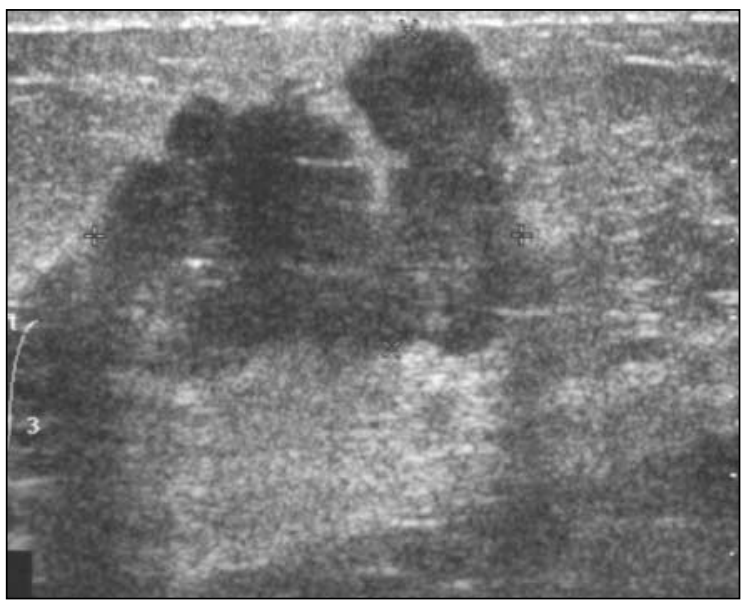

A

Figure 3. Histologic complete response: change in gray scale appearance after chemotherapy. A, Before chemotherapy, the breast cancer is an irregular hypoechoic mass with increased through-transmission. B, After chemotherapy, there is a smaller hypoechoic mass of a similar shape, now with shadowing.

development of sensitive and specific imaging methods to evaluate residual disease could be helpful for oncologists to spare patients with a complete histologic response from surgery, to plan surgical excision, or to determine whether more or alternative chemotherapy is needed. ${ }^{1}$

A

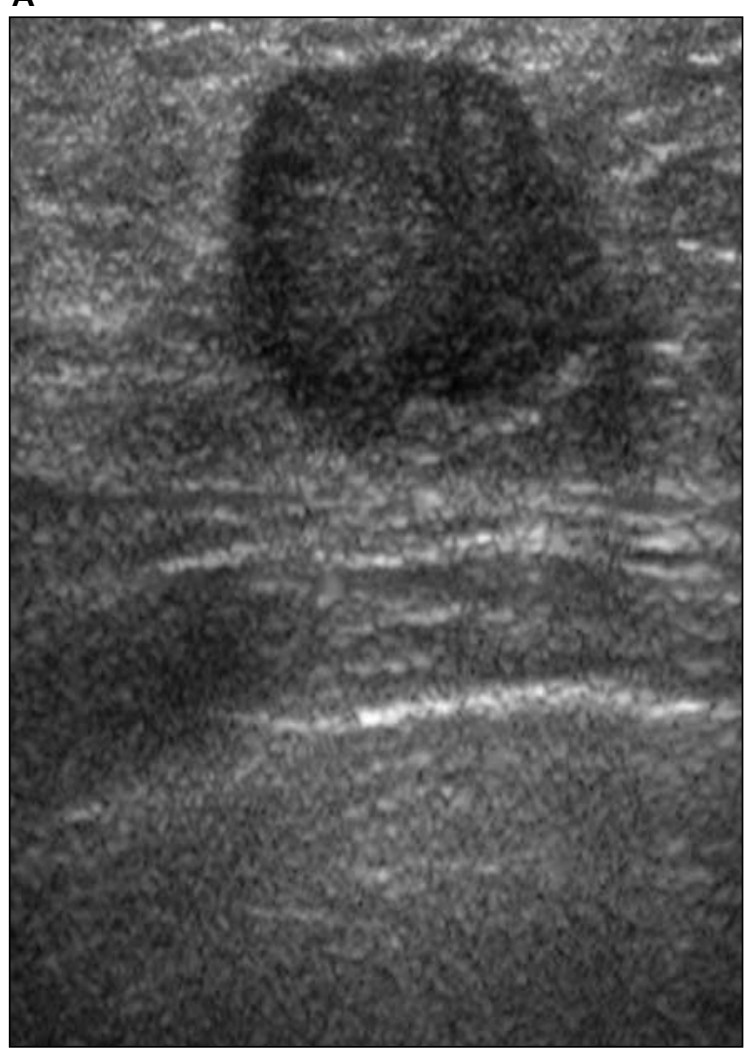

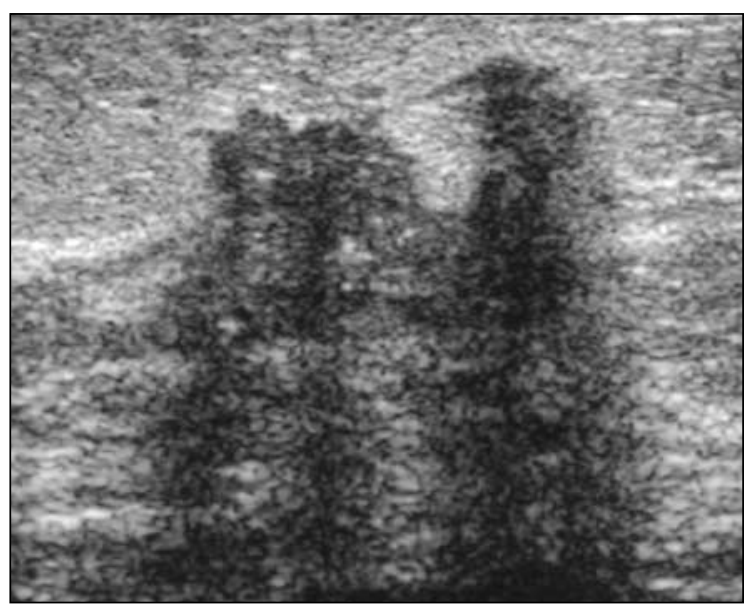

B
Physical examination and mammography have reproducibility and sensitivity limitations in assessing tumor response.,7 There are several research studies that have evaluated whether various imaging studies can show tumor responsiveness of locally advanced breast cancers to neoadjuvant chemotherapy. We studied patients with early-stage cancers because a neoadjuvant research protocol at our institution made this treatment available to patients with tumors as small as stage T1. Such a group facilitates detailed sonographic analyses compared with patients with large locally advanced tumors.

Figure 4. Change in gray scale appearance after chemotherapy. A, Before chemotherapy, the breast cancer is a circumscribed hypoechoic round mass. B, After chemotherapy, there is a tubular flat hypoechoic mass, which was residual cancer.

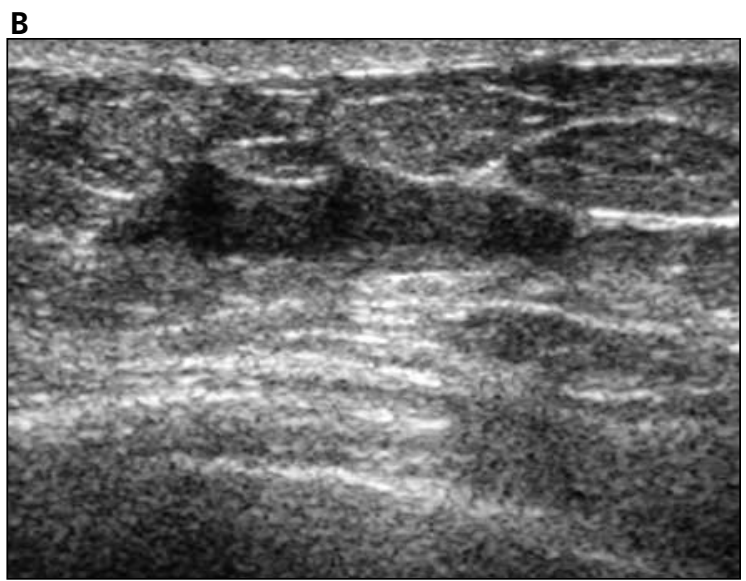


Table 2. Mean Vascularity of Masses as Measured by SWD

\begin{tabular}{lccc}
\hline Result & $\begin{array}{c}\text { Before } \\
\text { Chemotherapy }\end{array}$ & $\begin{array}{c}\text { After } \\
\text { Chemotherapy }\end{array}$ & $\boldsymbol{P}^{*}$ \\
\hline True-positive $(n=27)$ & 0.0150 & 0.0082 & .03 \\
False-positive $(n=3)$ & 0.0042 & 0.0014 & .22 \\
False-negative $(n=4)$ & 0.0006 & NA & NA \\
\hline
\end{tabular}

NA indicates not applicable.

*Student $t$ test.
For example, some were performed with more than 1 sonography machine and more than 1 sonographer or radiologist. In addition, unlike our study using whole-volume images, 2dimensional static images were obtained. Color Doppler sonography was performed in only 1 study, and some radiologists were not blinded to other clinical or imaging results. Our correlation coefficient and estimations of residual tumor are comparable with those reported for MRI. ${ }^{17,19}$ The correlation between imaging results and histologic results after neoadjuvant chemotherapy is probably affected by the type of chemotherapy. Tumor shrinkage in a docetaxel treatment group is reported to result in multiple microscopic nests of tumors rather than a single nodular lesion. ${ }^{20}$ This effect of docetaxel may also explain the false-negative results in our study. Comparison of tumor size between

Figure 5. Decrease in color flow Doppler signal and change in gray scale features after chemotherapy. A and B, Before chemotherapy, the breast cancer appears as a hypoechoic circumscribed lobulated mass with some posterior acoustic enhancement and a profuse color Doppler signal within it and adjacent to it. C, After chemotherapy, there is an indistinctly margined hypoechoic mass with some shadowing. The color Doppler signal was absent in the mass and was decreased in the tissue adjacent to the mass.

A

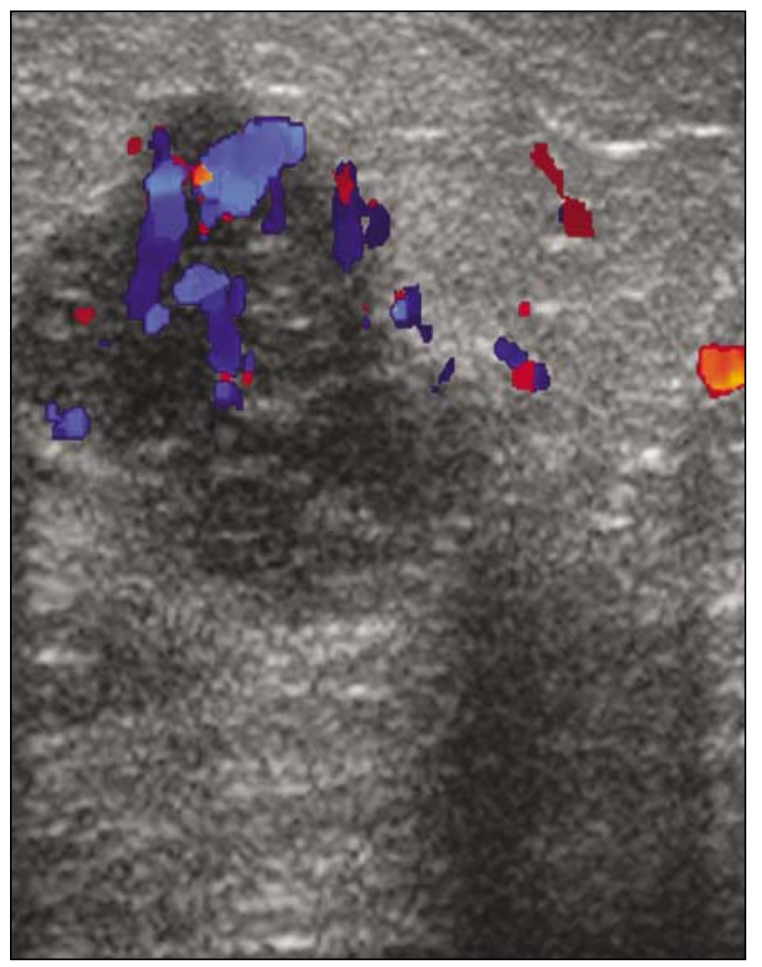

B

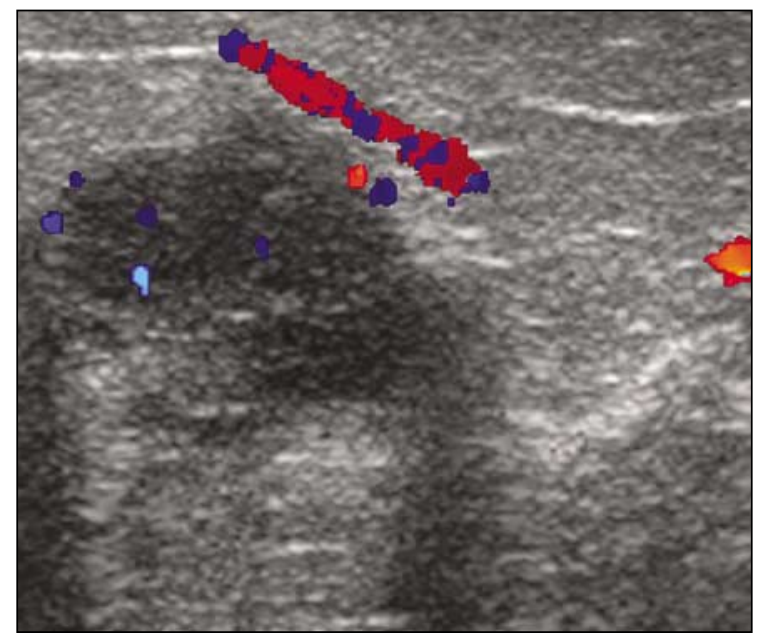

C

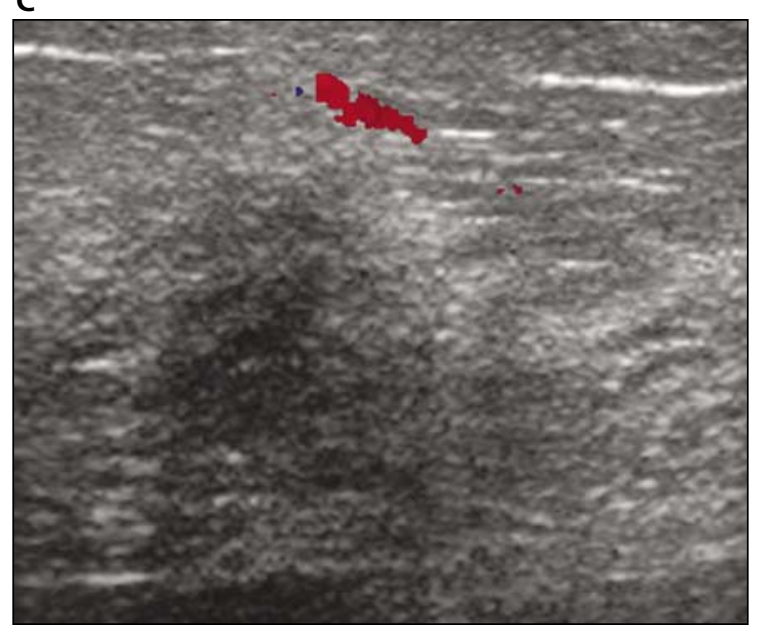


sonography and histologic examination may be more difficult after chemotherapy because tumors may become smaller and multifocal, making them less discrete on sonography and harder to measure at both sonography and histologic examination. Forouhi et $\mathrm{al}^{3}$ reported that sonography was superior to clinical examination in measuring breast tumor size after chemotherapy and reported a higher correlation coefficient, 0.89 . This higher correlation coefficient is likely due to the systematic processing of histologic specimens in their study, wherein size was measured from serial thin sections of fresh resected specimens. In our study group, sonography was sensitive in detecting residual tumors of $7 \mathrm{~mm}$ or larger. However, sonography was not able to distinguish between fibrosis, biopsy-related changes, and residual cancer. Furthermore, chemotherapy-induced histologic changes may contribute to the less than perfect correlation between the sonographic measurement of final maximum tumor size and the histologic measurement. ${ }^{21}$ For example, fibrous tissue is a common histologic finding in tumor sites after neoadjuvant chemotherapy, ${ }^{22,23}$ which can cause a visible mass on sonography and may cause the sonographic volume to be larger than the histologic volume of residual cancer. ${ }^{23}$

Color Doppler sonography has been shown to show neovascular characteristics in breast cancers, and some authors suggested that it may have the potential to measure tumor response. ${ }^{18}$ Huber et $\mathrm{al}^{18}$ studied color Doppler sonography in locally advanced breast cancers and found high correlation between histologic results and semiquantitative color Doppler results, with 1 patient who had a complete response having absent vascularity in a mass (fibrosis). A limitation in these previous studies as well as our own study was the small proportion of patients $(10 \%-15 \%)$ who completely responded to the neoadjuvant chemotherapy. In our study, there were 9 residual cancers up to $2.5 \mathrm{~cm}$ in size that had a sonographic mass without any vascularity. Therefore, the finding of a mass without vascularity was not specific for a complete tumor response in our study group. The vascularity measures decreased in most of our cases after chemotherapy but were not useful to indicate whether a complete response had occurred. The pathophysiologic explanation for the decrease in vascularity in these cases was not studied and remains unknown. It could possibly be related to the decrease in tumor size during chemotherapy, chemotherapy's effect on angiogenesis, or both. Differences between our study and the report by Huber et $\mathrm{al}^{18}$ about the usefulness of color Doppler sonography in assessment of residual cancer may be due to chemotherapy regimens, with taxanes not previously studied with color Doppler sonography. Docetaxel may have antiangiogenic activity, which may be a reason for the decrease in the vascular measures that occurred in most of our cases, an effect that was too common to indicate a complete histologic response. ${ }^{24}$ It has been suggested that hypervascularity and hypermetabolic activity might be indicators of the likelihood of tumor response. This is based on the hypothesis that tumors with poor perfusion may not receive adequate delivery of systemic therapy or that underperfused tumors might be hypoxic. ${ }^{8,25}$ Hypoxia has been

Table 3. Vascularity of Masses Determined by BIRADS Vascularity Classifications

\begin{tabular}{|c|c|c|c|}
\hline $\begin{array}{l}\text { BIRADS Vascularity } \\
\text { Classification }\end{array}$ & $\begin{array}{c}\text { Before } \\
\text { Chemotherapy }\end{array}$ & $\begin{array}{c}\text { After } \\
\text { Chemotherapy }\end{array}$ & $P^{*}$ \\
\hline \multicolumn{4}{|l|}{ Entire group $(n=34)$} \\
\hline Not present, n (\%) & $7 / 34(21)$ & $17 / 34(50)$ & .05 \\
\hline Increased in surrounding tissue, $\mathrm{n}(\%)$ & $7 / 34(21)$ & $7 / 34(21)$ & NS \\
\hline Present adjacent to lesion, $\mathrm{n}(\%)$ & $23 / 34(68)$ & $10 / 30(33) \dagger$ & .009 \\
\hline Present in lesion, $\mathrm{n}(\%)$ & $11 / 34(32)$ & $3 / 30(10) \dagger$ & .06 \\
\hline \multicolumn{4}{|l|}{ Subgroup of complete responders $(n=3)$} \\
\hline Not present, n (\%) & $1(33)$ & $2(67)$ & NS \\
\hline Increased in surrounding tissue, $\mathrm{n}$ & 0 & 0 & NS \\
\hline Present adjacent to lesion, $\mathrm{n}(\%)$ & $2(67)$ & $1(33)$ & NS \\
\hline Present in lesion, $\mathrm{n}$ & 0 & 0 & NS \\
\hline
\end{tabular}

NS indicates not significant.

* McNemar test.

tFour cases did not have a mass after chemotherapy. 
associated with aggressive tumor behavior and poor tumor responsiveness to chemotherapy. ${ }^{8,25}$ Contrary to this hypothesis, in our study group, high vascularity at initial imaging did not predict tumor response, and the complete responders had comparatively low vascularity.

To our knowledge, there are no previous studies that have evaluated whether any imaging characteristics at initial imaging can predict the response to chemotherapy. We did not find that any gray scale or vascular characteristic predicted tumor response, although the statistical evaluation was limited by the small numbers of complete responders. We also found that gray scale characteristics commonly change after chemotherapy but not in a predictable way. Some changes may be due to shrinking tumor size, development of fibrosis or cellular necrosis, and the tumor's becoming multifocal. Therefore, the appearance of the tumor after chemotherapy may be rather different, and identifying the location with tissue markers is important for postchemotherapy localization. Gray scale characteristics also do not distinguish the false-positive results from the true-positive residual tumors. A limitation of our study was that tumors were histologically fixed in the usual way, and paraffin fixation might alter tumor size. Because pathologic evaluation of tumor size remains the standard for evaluation of a residual tumor after chemotherapy, there may be a role for development of new histologic mapping of the tumor bed to more precisely compare imaging studies in these patients. ${ }^{21}$ Another limitation of our study is that tumors were not analyzed for microvessel density; hence, we cannot compare the color Doppler results with histologic vascularity.

In summary, we found that, after chemotherapy, the maximum tumor size at final histologic examination correlated quite well with the final sonographic size among cancers that were $7 \mathrm{~mm}$ or larger in maximal size. However, sonographic imaging yielded false-negative results in detecting residual tumors that were smaller than this threshold size. On the basis of our findings and those of other studies, we hypothesize that the histologic effects of docetaxel may contribute to false-negative gray scale sonographic results, and that antiangiogenic effects make a decrease in vascularity a nonspecific common finding. The presence of a mass after treatment may or may not signify a viable tumor because fibrosis can falsely mimic a tumor, and no gray scale or vas- cular characteristic was useful to distinguish these fibroses from residual tumors. This means that postchemotherapy biopsy would be necessary to determine with confidence whether a residual tumor was present. Gray scale features often change, and the appearance of the mass after chemotherapy may be quite different compared with initial imaging.

Sonography has advantages for assessment of breast cancers that are treated with neoadjuvant chemotherapy. Compared with mammography, evaluation of the breast by sonography is not limited by mammogram density, and nearly all cancers are evident at initial imaging. Compared with MRI, all patients are able to undergo sonographic evaluation; sonographically guided biopsies are simpler for assessing chemotherapy response; and it is a relatively low-cost modality. Radiologists and sonographers who perform breast sonography should be aware of the changing appearance of breast cancers during chemotherapy and of the advantages and limitations of sonography in assessing tumor response.

\section{References}

1. Green M, Hortobagyi GN. Neoadjuvant chemotherapy for operable breast cancer. Oncology 2002; 16:871-884.

2. Ganem G, Tubiana-Hulin M, Fumoleau P, et al. Phase II trial combining docetaxel and doxorubicin as neoadjuvant chemotherapy in patients with operable breast cancer. Ann Oncol 2003; 14:1623-1628.

3. Forouhi P, Walsh JS, Anderson TJ, Chetty U. Ultrasonography as a method of measuring breast tumour size and monitoring response to primary systemic treatment. Br J Surg 1994; 81:223-225.

4. Balu-Maestro C, Chapellier C, Bleuse A, Chanalet I, Chauvel C, Largillier R. Imaging in evaluation of response to neoadjuvant breast cancer treatment benefits of MRI. Breast Cancer Res Treat 2002; 72:145-152.

5. Warren RM, Bobrow LG, Earl HM, et al. Can breast MRI help in the management of women with breast cancer treated by neoadjuvant chemotherapy? Br J Cancer 2004; 90:1349-1360.

6. Golshan M, Fung BB, Wiley E, Wolfman J, Rademaker A, Morrow M. Prediction of breast cancer size by ultrasound, mammography and core biopsy. Breast 2004; 13:265-271. 
7. Helvie MA, Joynt LK, Cody RL, Pierce $\sqcup$, Adler DD, Merajver SD. Locally advanced breast carcinoma: accuracy of mammography versus clinical examination in the prediction of residual disease after chemotherapy. Radiology 1996; 198:327-332.

8. Mankoff DA, Dunnwald LK, Gralow JR, et al. Blood flow and metabolism in locally advanced breast cancer: relationship to response to therapy. J Nucl Med 2002; 43:500-509.

9. Bhatti PT, LeCarpentier GL, Roubidoux MA, Fowlkes JB, Helvie MA, Carson PL. Discrimination of sonographically detected breast masses using frequency shift color Doppler imaging in combination with age and gray scale criteria. J Ultrasound Med 2001; 20: 343-350.

10. LeCarpentier GL, Tridandapani P, Fowlkes JB, Roubidoux MA, Moskalik AP, Carson PL. Utility of $3 \mathrm{D}$ ultrasound in the discrimination and detection of breast cancer. RSNA EJ 1999. Available at: http://ej.rsna.org/ej3/0103-99.fin/titlepage.html.

11. American College of Radiology. ACR Practice Guideline for the Performance of Ultrasound-Guided Percutaneous Breast Interventional Procedures. Reston, VA: American College of Radiology; 2003.

12. Chang J, Powles TJ, Allred DC, et al. Biologic markers as predictors of clinical outcome from systemic therapy for primary operable breast cancer. J Clin Oncol 1999; 17:3058-3063.

13. Fornage BD, Toubas O, Morel M. Clinical, mammographic, and sonographic determination of preoperative breast cancer size. Cancer 1987; 60:765-771.

14. Yang WT, Lam WW, Cheung H, Suen M, King WW, Metreweli C. Sonographic, magnetic resonance imaging, and mammographic assessments of preoperative size of breast cancer. J Ultrasound Med 1997; 16:791-797.

15. Hieken TJ, Harrison J, Herreros J, Velasco JM. Correlating sonography, mammography, and pathology in the assessment of breast cancer size. Am J Surg 2001; 182:351-354.

16. Zeggelink WF, Deurloo EE, Bartelink H, Rutgers EJ, Gilhuijs KG. Reproducibility of the assessment of tumor extent in the breast using multiple image modalities. Med Phys 2003; 30:2919-2926.

17. Londero $V$, Bazzocchi M, Del Frate $C$, et al. Locally advanced breast cancer: comparison of mammog- raphy, sonography and MR imaging in evaluation of residual disease in women receiving neoadjuvant chemotherapy. Eur Radiol 2004; 14:1371-1379.

18. Huber S, Medl M, Helbich T, et al. Locally advanced breast carcinoma: computer assisted semiquantitative analysis of color Doppler ultrasonography in the evaluation of tumor response to neoadjuvant chemotherapy. J Ultrasound Med 2000; 19:601607.

19. Rosen EL, Blackwell KL, Baker JA, et al. Accuracy of $\mathrm{MRI}$ in the detection of residual breast cancer after neoadjuvant chemotherapy. AJR Am J Roentgenol 2003; 181:1275-1282.

20. Denis F, Desbiez-Bourcier AV, Chapiron C, Arbion F, Body G, Brunereau L. Contrast enhanced magnetic resonance imaging underestimates residual disease following neoadjuvant docetaxel based chemotherapy for breast cancer. Eur J Surg Oncol 2004; 30: 1069-1076.

21. Rajan R, Esteva FJ, Symmans WF. Pathologic changes in breast cancer following neoadjuvant chemotherapy: implications for the assessment of response. Clin Breast Cancer 2004; 5:235-238.

22. Honkoop AH, Pinedo HM, De Jong JS, et al. Effects of chemotherapy on pathologic and biologic characteristics of locally advanced breast cancer. Am J Clin Pathol 1997; 107:211-218.

23. Anderson TJ, Dixon JM, Stuart M, Sahmoud T, Miller WR. Effect of neoadjuvant treatment with anastrozole on tumour histology in postmenopausal women with large operable breast cancer. $\mathrm{Br} J$ Cancer 2002; 87:334-338.

24. Guo $X L$, Lin GJ, Zhao $H$, et al. Inhibitory effects of docetaxel on expression of VEGF, bFGF and MMPs of LS174T cell. World J Gastroenterol 2003; 9:1995-1998.

25. Mankoff DA, Dunnwald LK, Gralow JR, et al. Changes in blood flow and metabolism in locally advanced breast cancer treated with neoadjuvant chemotherapy. J Nucl Med 2003; 44:1806-1814. 